\title{
Article
}

\section{Gut Microbiota Prevents Sugar Alcohol-Induced Diarrhea}

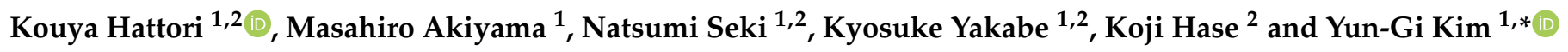 \\ 1 Research Center for Drug Discovery, Faculty of Pharmacy and Graduate School of Pharmaceutical Sciences, \\ Keio University, Tokyo 105-8512, Japan; kouya19950425@keio.jp (K.H.); akiyama.masahiro@keio.jp (M.A.); \\ natsumisoshiru826@keio.jp (N.S.); kkkeeoosuyi@keio.jp (K.Y.) \\ 2 Department of Biochemistry, Faculty of Pharmacy and Graduate School of Pharmaceutical Sciences, \\ Keio University, Tokyo 105-8512, Japan; hase-kj@pha.keio.ac.jp \\ * Correspondence: ykim@keio.jp
}

check for

updates

Citation: Hattori, K.; Akiyama, M.; Seki, N.; Yakabe, K.; Hase, K.; Kim, Y.-G. Gut Microbiota Prevents Sugar Alcohol-Induced Diarrhea. Nutrients 2021, 13, 2029. https://doi.org/ $10.3390 /$ nu13062029

Academic Editors: Eva Untersmayr and Peter M. Abuja

Received: 29 April 2021

Accepted: 10 June 2021

Published: 12 June 2021

Publisher's Note: MDPI stays neutral with regard to jurisdictional claims in published maps and institutional affiliations.

Copyright: (c) 2021 by the authors. Licensee MDPI, Basel, Switzerland. This article is an open access article distributed under the terms and conditions of the Creative Commons Attribution (CC BY) license (https:/ / creativecommons.org/licenses/by/ $4.0 /)$.

\begin{abstract}
While poorly-absorbed sugar alcohols such as sorbitol are widely used as sweeteners, they may induce diarrhea in some individuals. However, the factors which determine an individual's susceptibility to sugar alcohol-induced diarrhea remain unknown. Here, we show that specific gut bacteria are involved in the suppression of sorbitol-induced diarrhea. Based on 16S rDNA analysis, the abundance of Enterobacteriaceae bacteria increased in response to sorbitol consumption. We found that Escherichia coli of the family Enterobacteriaceae degraded sorbitol and suppressed sorbitol-induced diarrhea. Finally, we showed that the metabolism of sorbitol by the E. coli sugar phosphotransferase system helped suppress sorbitol-induced diarrhea. Therefore, gut microbiota prevented sugar alcohol-induced diarrhea by degrading sorbitol in the gut. The identification of the gut bacteria which respond to and degrade sugar alcohols in the intestine has implications for microbiome science, processed food science, and public health.
\end{abstract}

Keywords: gut microbiota; sugar alcohol; diarrhea

\section{Introduction}

Sugar alcohols such as sorbitol, mannitol, and xylitol are naturally present in some fruits, vegetables, and mushrooms but are classified as artificial sweeteners since they can be industrially produced by reducing saccharides such as glucose [1,2]. These sugar alcohols have a low caloric content due to poor absorption in the small intestine and are thus frequently used as sweeteners in "sugar-free" candy, chewing gum, and beverages [3]. Because of their stability and resistance to heat, they are also used for a wide variety of other purposes, including as food moisturizing agents, preservatives, and excipients. However, ingestion of these poorly-absorbed sugar alcohols may lead to gastrointestinal symptoms in some people and have laxative effects. For example, it has been reported that the excessive use of chewing gum containing sorbitol may cause symptoms such as diarrhea, weight loss, and bloating $[4,5]$. The main cause of the diarrhea is thought to be the accumulation of poorly-absorbed sugar alcohols in the colon, where they increase colonic osmotic pressure and prevent water absorption [6]. Interestingly, susceptibility to sugar alcohol-induced diarrhea is known to vary among individuals [6]. However, the mechanisms underlying these effects remain unknown.

Poorly-absorbed carbohydrates can be a source of energy for some bacteria residing in the gut [7]. The human gut contains approximately 40 trillion bacteria that form complex communities of which the composition varies among individuals due to differences in diet, lifestyle, and antibiotic use [8,9]. Consumption of poorly-absorbed carbohydrates is one factor that can alter the composition of the gut microbiota. Because the carbohydrates preferred by gut bacteria vary from species to species, the ingestion of poorly-absorbed carbohydrates with different chemical structures can promote the development of a distinctive gut flora [10]. Additionally, sugar alcohols like sorbitol, which are a type of poorly-absorbed 
carbohydrate, are known to be degraded by certain bacteria [11,12]. However, the relationship between the consumption of poorly-absorbed sugar alcohols and their biological effects, such as osmotic diarrhea and gut bacteria makeup, remains poorly understood.

We hypothesized that sugar alcohol-degrading bacteria exist in the intestine and also that susceptibility to sugar alcohol-induced diarrhea depends on the amount of these bacteria being present. When the intestine contains low amounts of sugar alcohol-degrading bacteria, sugar alcohols accumulate, which leads to increased osmotic pressure and diarrhea. In the current study, we investigated the relationship between sugar alcohol-induced diarrhea and gut microbiota in an effort to identify the bacteria responsible for the prevention of diarrhea symptoms.

\section{Materials and Methods}

\subsection{Mice}

Male C57BL/6 mice were purchased from the Sankyo Labo Service Corporation (Tokyo, Japan) and were kept under conventional conditions. Male MCH (ICR) mice, germ-free (GF) male ICR mice, and ICR-derived inbred strain IQI mice were purchased from CLEA Japan, Inc. (Tokyo, Japan). GF male MCH and IQI mice were maintained in vinyl isolators. Male $\mathrm{MCH}$ mice were kept under specific pathogen-free (SPF) conditions. These mice were fed $\gamma$-ray-sterilized AIN-93G (Oriental Yeast, Tokyo, Japan). In order to evaluate the effect of sugar alcohol on diarrhea, mice were administered water drinking water containing $5 \%$ or $10 \%$ sorbitol, or $5 \%$ mannitol for 4 days. For experiments involving antibiotics, mice were administered drinking water containing ampicillin $(1 \mathrm{~g} / \mathrm{L})$, streptomycin $(5 \mathrm{~g} / \mathrm{L})$, erythromycin $(200 \mathrm{mg} / \mathrm{L})$, or vancomycin $(250 \mathrm{mg} / \mathrm{L})$. For gnotobiotic experiments, GF male ICR or IQI mice (obtained from CLEA Japan) were bred in vinyl isolators and fed $\gamma$-ray-sterilized CMF. During the experiment, GF mice were fed a $\gamma$-ray-sterilized AIN-93G.

All mice were housed under $21-22{ }^{\circ} \mathrm{C}$ with a $12 \mathrm{~h}$ alternating light-dark cycle at the animal facilities of Faculty of Pharmacy, Keio University (Tokyo, Japan). All animal experiments were performed according to the Institutional Guidelines for the Care and Use of Laboratory Animals in Research and were approved by the local ethics committees at Keio University.

\subsection{Bacteria}

Bacteria isolated from mouse feces and strains purchased from the Japan Collection of Microorganisms (JCM, Ibaraki, Japan) were used in the study. Wild-type (WT) and gene mutants ( $s r l A-$, srlB-, srlE-, or srlD) of E. coli K-12 were purchased from the National Bio Resource Project (NBRP, Shizuoka, Japan).

\subsection{Colonization of Germ-Free Mice}

Escherichia coli and Proteus mirabilis isolated from the stool of C57BL/6 mice, or WT and $\operatorname{srlD}$ mutant of $E$. coli $\mathrm{K}-12$ were used for the experiments. GF male IQI or ICR mice were colonized with $200 \mu \mathrm{L}$ of bacterial culture via oral gavage.

\subsection{Evaluation of Diarrhea Symptoms}

The severity of diarrhea was evaluated based on body weight change and fecal water content after the sugar alcohol treatment. Body weight change was recorded daily in the morning. The collected feces were weighed, dried overnight in an incubator, and re-weighed. The fecal water content was calculated from the weight before drying and the weight after drying.

\section{5. $16 S$ rRNA Gene Sequencing and Analysis}

Approximately $200 \mathrm{mg}$ of each stool sample was transferred into $2 \mathrm{~mL}$ tubes containing $0.1 \mathrm{~mm}$ zirconia/silica beads and $3.0 \mathrm{~mm}$ zirconia beads. The stool samples were homogenized for $10 \mathrm{~min}$ in a Shake Master Neo cell disruption device (Biomedical Sciences, 
Tokyo, Japan) after the addition of $540 \mu \mathrm{L}$ of SLX-Mlus Buffer from an E.Z.N.A. Stool DNA Kit (Omega Bio-tek, Norcross, GA, USA). After homogenization, $60 \mu \mathrm{L}$ of DS buffer and $20 \mu \mathrm{L}$ of Proteinase K solution from the E.Z.N.A. Stool DNA Kit were added. The samples were then incubated at $70{ }^{\circ} \mathrm{C}$ for $10 \mathrm{~min}$ and at $95^{\circ} \mathrm{C}$ for $5 \mathrm{~min}$. SP2 buffer from the E.Z.N.A. Stool DNA Kit was then added and the samples were incubated on ice for $5 \mathrm{~min}$. The samples were centrifuged at $13,000 \times g$ for 5 min and DNA was extracted from $200 \mu \mathrm{L}$ of the supernatant using a magLEAD $12 \mathrm{gC}$ nucleic acid extraction instrument (Precision System Science, Chiba, Japan) with a MagLEAD Consumable Kit and MagDEA ${ }^{\circledR}$ Dx SV. The extracted genomic DNA was resuspended at $5 \mathrm{ng} / \mu \mathrm{L}$ in $10 \mathrm{mM}$ Tris- $\mathrm{HCl}$ buffer. The DNA was used to prepare $16 \mathrm{~S}$ rRNA gene libraries in accordance with the protocol described in an Illumina technical note (https: / / support.illumina.com/documents / documentation/ chemistry_documentation/16s/16s-metagenomic-library-prep-guide-15044223-b.pdf (accessed on 9 May 2019). Briefly, each DNA sample was amplified by polymerase chain reaction (PCR) using KAPA HiFi HS ReadyMix (Nippon Genetics, Tokyo, Japan) and primers specific for variable regions 3 and 4 of the 16S rRNA gene. The PCR products were purified using AMPure XP Beads (Beckman Coulter, Brea, CA, USA) and adapters added by PCR using a Nextera XT Index Kit. The libraries were further purified using AMPure XP Beads, diluted to $10 \mathrm{nM}$ with $10 \mathrm{mM}$ Tris- $\mathrm{HCl}$ buffer, and pooled. The pooled samples were sequenced using a MiSeq System (Illumina, San Diego, CA, USA) with a $2 \times 300$-base-pair protocol. Sequences were analyzed using QIIME (v. 1.9.1) software (http:/ / qiime.org, accessed on 10 June 2021) [13]. Reads with an average quality value $<20$ were excluded using Trimmomatic (v. 0.36) software (http:/ / www.usadellab.org/cms/?page=trimmomatic, accessed on 10 June 2021) [14]. Paired-end sequences were joined using a Fastq-join tool in the EA-Utils software package (https:/ / expressionanalysis.github.io/ea-utils/, https: / / openbioinformaticsjournal.com/VOLUME/7/PAGE/1/, accessed on 10 June 2021). High-quality sequences $(11,000)$ were randomly chosen from the quality filter-passed sequences that were obtained for each sample. After trimming both primer sequences using cutadapt (https:/ / doi.org/10.14806/ ej.17.1.200, accessed on 10 June 2021), chimeras were detected using the USEARCH de novo method. The sequences were assigned to operational taxonomic units (OTUs) using the UCLUST algorithm with a sequence identity threshold of $96 \%$. Taxonomic assignments for each OTU were made by similarity searching against publicly available $16 \mathrm{~S}$ sequences (RDP v. 10.27 (The Center for Microbial Ecology, East Lansing, Michigan, US) and CORE update 2 September 2012) and NCBI genome databases using the blastn program available from the local Basic Local Alignment Search Tool (BLAST). The data were simplified to 5000-10,000 sequences per sample using rarefaction curves, and the relative abundances of the community members were determined using the rarefied data. The analysis was performed as described in a previous report [15]. Principal coordinate analysis (PCoA) was used to analyze the beta diversity using an unweighted UniFrac metric calculated by QIIME. Permutational multivariate analysis of the variance (PERMANOVA) test was performed using the vegan package (v 2.7) (https:/ / github.com/vegandevs/vegan, accessed on 10 June 2021) in R.

\subsection{DNA Extraction and Quantitative PCR ( $q P C R$ )}

Bacterial DNA was isolated from $20 \mathrm{mg}$ of fecal samples (or $200 \mu \mathrm{L}$ of standard culture). Extraction and quantification of the bacteria DNA were conducted as described in a previous report [16]. Briefly, a 20-fold diluted fecal sample $(200 \mu \mathrm{L})$ was mixed with $300 \mu \mathrm{L}$ of extraction buffer (100 mM Tris-HCl, 40 mM EDTA, 1.7\% SDS, pH 9.0), $500 \mu \mathrm{L}$ of buffer-saturated phenol, and $300 \mathrm{mg}$ of glass beads. The mixture was vortexed vigorously for $15 \mathrm{~s}$ at $5000 \mathrm{rpm} 3$ times using a Precellys 24 homogenizer (M\&S Instruments Inc., Osaka, Japan). After centrifugation at $14,000 \times g$ for $5 \mathrm{~min}, 400 \mu \mathrm{L}$ of the supernatant was collected. Phenol-chloroform extractions were subsequently performed and $250 \mu \mathrm{L}$ of the supernatant was subjected to isopropanol precipitation. Finally, the DNA was suspended in $1 \mathrm{~mL}$ of TE buffer. 
The total number of bacteria in the feces was analyzed by qPCR using universal primers. PCR amplification and detection were performed using a CFX-96 Real-Time system (Bio-Rad, Hercules, CA, USA). Each reaction mixture $(20 \mu \mathrm{L})$ consisted of $10 \mu \mathrm{L}$ of SYBR premix Ex Taq II (Takara, Shiga, Japan), $0.4 \mu \mathrm{L}$ of each primer $(10 \mu \mathrm{M}), 2 \mu \mathrm{L}$ of DNA template, and $7.2 \mu \mathrm{L}$ of distilled water. The amplification profile consisted of one cycle at $95{ }^{\circ} \mathrm{C}$ for $30 \mathrm{~s}$ followed by 35 cycles of $95^{\circ} \mathrm{C}$ for $5 \mathrm{~s}, 60{ }^{\circ} \mathrm{C}$ for $30 \mathrm{~s}$. The fluorescent signal of the amplified products was detected during the last step of each cycle. Melting curve analysis was performed after amplification to distinguish the targeted PCR product from non-targeted product. The melting curves were obtained by slow heating the samples from $65{ }^{\circ} \mathrm{C}$ to $95^{\circ} \mathrm{C}$ at a rate $0.5^{\circ} \mathrm{C} / \mathrm{s}$.

\subsection{Isolation and Identification of Bacteria from Feces}

Fecal samples were diluted with phosphate-buffered saline (PBS) and applied to Brain Heart Infusion agar (BD, Franklin Lakes, NJ, Japan) and modified Gifu Anaerobic Medium agar (Nissui, Tokyo, Japan) and cultured under anaerobic conditions. After $24 \mathrm{~h}$ to $72 \mathrm{~h}$ culture, some colonies were picked and the isolated colonies lysed in $100 \mu \mathrm{L}$ of $50 \mathrm{mM}$ $\mathrm{NaOH}$. After incubation at $95{ }^{\circ} \mathrm{C}$ for $10 \mathrm{~min}$, the lysed colonies were centrifuged at $3000 \times g$ for $10 \mathrm{~min}$. Then, $20 \mu \mathrm{L}$ of extracted solution was diluted with $100 \mu \mathrm{L}$ Tris- $\mathrm{HCl}$ (pH 7.0-7.2). The diluted DNA was used as template for PCR amplification. The PCR reaction mixtures $(25 \mu \mathrm{L})$ consisted of $18 \mu \mathrm{L}$ of KOD FX Neo reagent solution $(12.5 \mu \mathrm{L} 2 \times$ buffer, $5 \mu \mathrm{L}$ dNTPs, and $0.5 \mu \mathrm{L}$ enzyme solution), $0.75 \mu \mathrm{L}$ of each primer $(10 \mu \mathrm{M}), 2 \mu \mathrm{L}$ of DNA template, and $3.5 \mu \mathrm{L}$ of distilled water. The amplification profile consisted of one cycle at $94{ }^{\circ} \mathrm{C}$ for 2 min followed by 40 cycles of $98^{\circ} \mathrm{C}$ for $10 \mathrm{~s}, 55^{\circ} \mathrm{C}$ for $30 \mathrm{~s}$, and $68{ }^{\circ} \mathrm{C}$ for $30 \mathrm{~s}$ with a final extension step of $72{ }^{\circ} \mathrm{C}$ for $5 \mathrm{~min}$. After purification using ExoSAP-IT ExpressPCR Cleanup Reagents (ThermoFisher, Waltham, MA, Japan), the samples were sequenced using capillary sequence services from Hokkaido System Science Co., Ltd. (Sapporo, Hokkaido, Japan).

\subsection{Evaluation of Sorbitol Degradation and Measurement of Sorbitol Concentration in Culture Supernatant}

M9 medium [17] was used as a control medium to culture Enterobacteriaceae isolates. The other bacteria were cultured using the Gifu Anaerobic Medium (GAM) Semisolid without Dextrose (Nissui), prepared in water and filtered to remove the agar. When sugar was added to the media, the concentration was adjusted to $10 \mathrm{mg} / \mathrm{mL}$. Following incubation under anaerobic conditions, $10 \mu \mathrm{L}$ of the bacterial culture broth from the previous day was placed in each medium. Bacterial growth was evaluated based on absorbance at $600 \mathrm{~nm}$ or $570 \mathrm{~nm}$ that was measured using an Infinite 200 Pro plate reader (Tecan, Seestrasse, Switzerland). Sorbitol concentrations in the culture supernatants were measured using an EnzyChrom Sorbitol Assay Kit (BioAssay Systems, Hayward, CA, USA).

\subsection{Statistical Analysis}

Statistical analyses were performed using R (version 3.6.3) (R Foundation, Viennam, Austria). For parametrical data when the variances were equal, we used Student's $t$-test for two groups, and one-way analysis of variance (ANOVA) followed by Dunnett's test (post-hoc test) for more than two groups, respectively. When the variances were unequal, we used Welch's $t$-test for two groups, and ANOVA followed by Steel's test (post-hoc test) for more than two groups, respectively. Differences with $p$-values $<0.05$ were considered statistically significant.

\section{Results}

\subsection{Specific Gut Microbiota Played a Protective Role against Sorbitol-Induced Diarrhea}

We first investigated the role of gut microbiota in sorbitol-induced diarrhea by comparing susceptibility to sorbitol-induced diarrhea in specific pathogen-free (SPF) mice, which have an intact gut microbiota, and germ-free (GF) mice, which lack a gut microbiota. 
Unlike that for the SPF mice, the body weight of the GF mice receiving sorbitol continued to decrease over the four-day experimental period and significant weight loss was observed at four days compared with that of the SPF mice (Figure 1A). GF mice, but not SPF mice, had increased fecal water content four days after receiving sorbitol (Figure 1B). These results indicated the gut microbiota played a protective role against sorbitol-induced diarrhea.

A

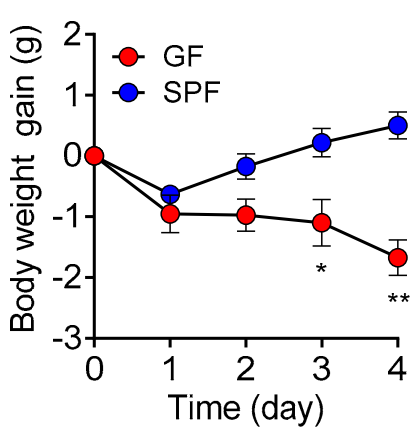

C

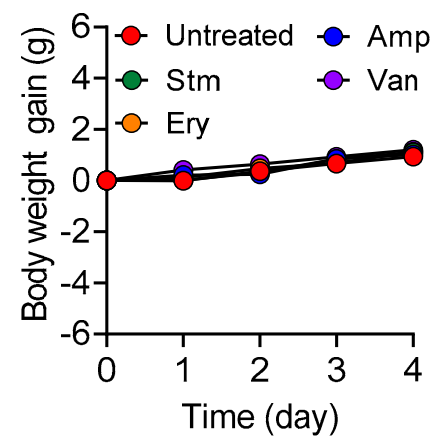

B

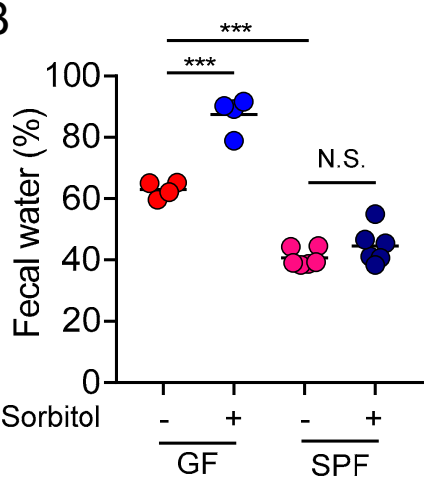

$\mathrm{D}$

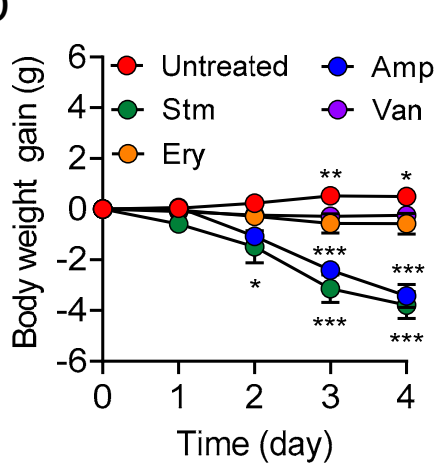

E

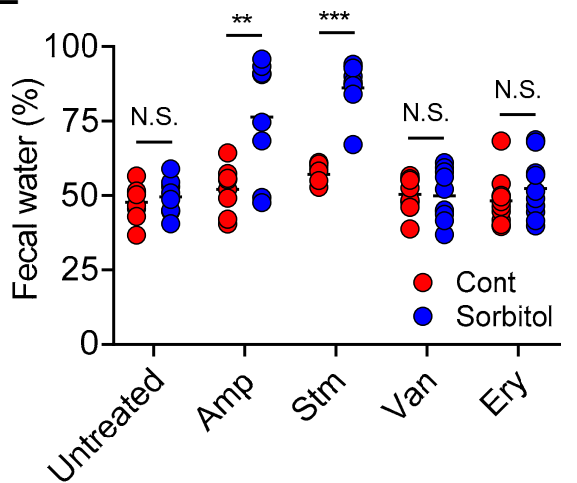

Figure 1. Specific Gut Microbiota Contributes to the Suppression of Sorbitol-Induced Diarrhea. (A,B) SPF and germ-free (GF) ICR mice were provided drinking water containing 5\% sorbitol for 4 days. (A) Body weight changes from day 0 to day 4 (SPF, $n=6$; GF, $n=4$ ). Data are mean \pm SEM. ${ }^{*} p<0.05,{ }^{* *} p<0.01$ compared to the day 0 weight of each group using Dunnett's test. (B) Fecal water content at day 4 . Each dot represents an individual mouse and the horizontal bars indicate mean values. N.S.; not significant, ${ }^{* * *} p<0.001$ by Tukey's multiple comparisons test. SPF, specific pathogen-free; GF, germ-free. (C-E) The C57BL/6J mice were provided drinking water with or without 5\% sorbitol for 4 days after antibiotic treatment as indicated. Cont, control; Sorbitol, 5\% sorbitol; Amp, ampicillin; Stm, streptomycin; Van, vancomycin; Ery, erythromycin. (C) Body weight changes from day 0 to day 4 during treatment with antibiotics. Data are mean \pm SEM. (D) Body weight changes from day 0 to day 4 during treatment with antibiotics and $5 \%$ sorbitol. Data are mean \pm SEM. ${ }^{*} p<0.05,{ }^{* *} p<0.01,{ }^{* * *} p<0.001$ compared to the day 0 weight of each group using Dunnett's test. (E) Fecal water content at day 0 and 4 after treatment with $5 \%$ sorbitol. Each dot represents an individual mouse and the horizontal bars indicate mean values. Data are from two independent experiments. N.S.; not significant, ${ }^{* *} p<0.01,{ }^{* * *} p<0.001$ by Welch's $t$-test.

We next investigated whether specific gut bacteria helped protect against sorbitolinduced diarrhea. To alter the composition of the gut microbiota, mice were treated with antibiotics that exhibit different antimicrobial spectra, and their susceptibility to sorbitolinduced diarrhea was examined. Treatment with antibiotics did not influence body weight change (Figure 1C). In the ampicillin-treated and streptomycin-treated mice, significant weight loss was observed after the administration of sorbitol compared with that in the antibiotic-free control group. These changes were not observed in the vancomycin-treated or erythromycin-treated mice (Figure 1D). Significant increases in sorbitol-induced fecal water content were observed in the ampicillin-treated and streptomycin-treated mice, but not in the vancomycin-treated or erythromycin-treated mice (Figure 1E). These results suggested vancomycin-resistant and erythromycin-resistant bacteria played protective 
roles against sorbitol-induced diarrhea. The involvement of gut bacteria in sugar alcoholinduced diarrhea other than sorbitol was confirmed based on mannitol-induced diarrhea, which was exacerbated by the administration of ampicillin (Figure S1). Overall, our results indicated that specific gut bacteria contributed to the inhibition of sugar alcoholinduced diarrhea.

\subsection{Enterobacteriaceae Were Identified as Gut Bacteria Responding to Sorbitol}

To identify the gut bacteria contributing to the suppression of sorbitol-induced diarrhea, we evaluated the composition of the gut microbiota of mice treated with antibiotics by $16 \mathrm{~S}$ rDNA analysis. The analysis confirmed that treatment with each of antibiotics altered the gut microbiota composition (Figure 2A), and revealed that bacteria of the orders Enterobacteriales and Clostridiales were the dominant bacteria in the vancomycin-treated and erythromycin-treated group, respectively (Figure 2B). Notably, the abundance of Enterobacteriales increased with the administration of increasing amounts of sorbitol in a concentration-dependent manner (Figure 2C,D). At the genus level, treatment with sorbitol and vancomycin resulted in the greatest increase in the abundance of Escherichia, Klebsiella, Enterobacter, and Proteus in the family Enterobacteriaceae (Figure 2E). In contrast, treatment with erythromycin increased the abundance of Lachnoclostridium in the family Clostridiaceae in the gut (Figure 2B and Figure S2). These results suggest that Enterobacteriaceae are one of the families of gut bacteria responding to sorbitol and may be involved in the suppression of sorbitol-induced diarrhea.

A

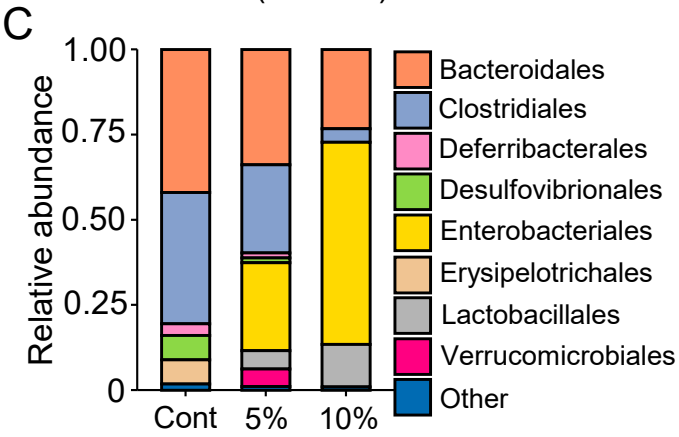

B

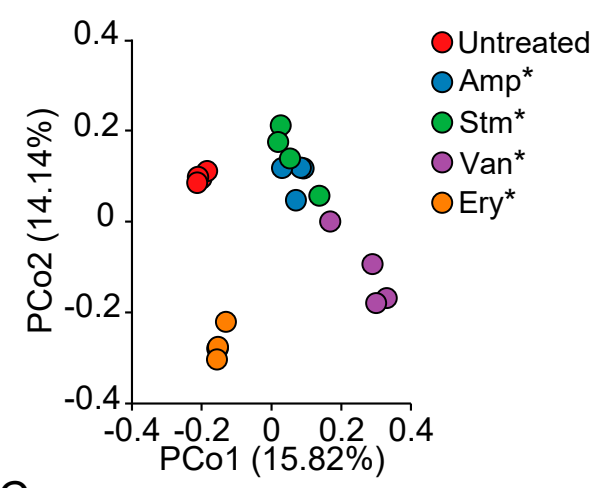

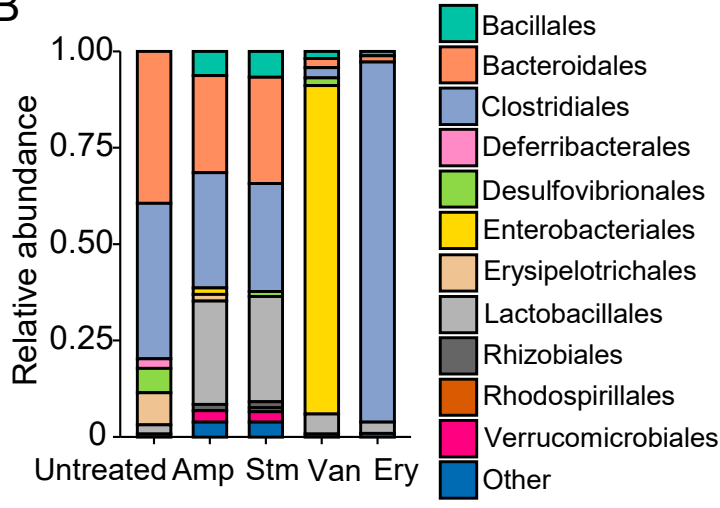

D

Figure 2. Cont. 


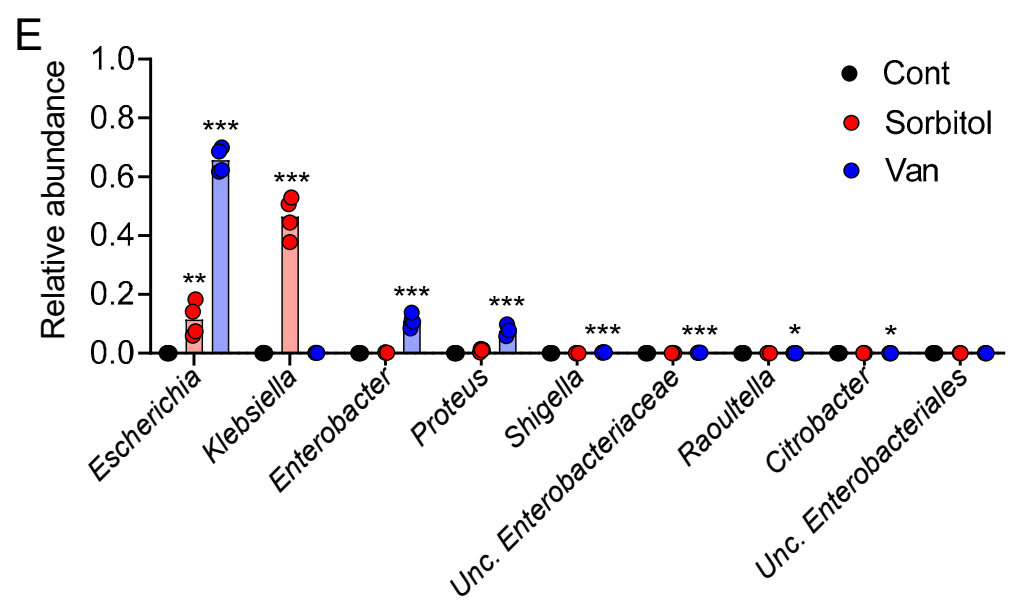

Figure 2. Identification of Enterobacteriaceae as Gut Bacteria Responding to Sorbitol. (A) Principal coordinate analysis (PCoA) plot generated using an unweighted UniFrac metric. Amp, ampicillin; Stm, streptomycin; Van, vancomycin; Ery, erythromycin. ${ }^{*} p<0.05$ by PERMANOVA $t$-test. (B) Relative abundance of operational taxonomic units (OTUs) in fecal samples from C57BL/6 J mice untreated or treated with antibiotics. The various colors correspond to each bacterial order indicated. Amp, ampicillin; Stm, streptomycin; Van, vancomycin; Ery, erythromycin. (C) Relative abundance of OTUs in fecal samples from sorbitol-treated C57BL/6J mice $(n=4)$. The various colors correspond to each bacterial order indicated. Cont, control; 5\%, 5\% sorbitol; $10 \%, 10 \%$ sorbitol. (D) Relative abundances of OTUs assigned to the family Enterobacteriaceae. Cont, control; 5\%, 5\% sorbitol; 10\%, 10\% sorbitol. Each dot represents an individual mouse and the horizontal bars indicate mean values. ${ }^{*} p<0.05,{ }^{* * *} p<0.001$ compared with the control group using Dunnett's $t$-test. (E) Relative abundances of OTUs assigned to the genera of the family Enterobacteriaceae. Bars in the graph are mean values. Each circle represents an individual mouse. ${ }^{*} p<0.05,{ }^{* *} p<0.01,{ }^{* * *} p<0.001$ compared to the Cont group using Steel's test. Cont, control; Amp, ampicillin; Stm, streptomycin; Van, vancomycin; Ery, erythromycin.

\subsection{E. coli Degraded Sorbitol and Suppressed Sorbitol-Induced Diarrhea}

To confirm that Enterobacteriaceae suppressed sorbitol-induced diarrhea by consuming sorbitol in the colon, we isolated Enterobacteriaceae bacteria which abundance was increased by sorbitol and/or vancomycin treatment, including E. coli, Citrobacter farmeri, Klebsiella pneumoniae, Enterobacter spp., and Proteus mirabilis, and assessed their ability to utilize sorbitol as a sole carbon source in culture medium. M9 media supplemented with $1 \%$ glucose or sorbitol were prepared and bacterial growth was measured based on absorbance. The growth of E. coli, C. farmeri, K. pneumoniae, and Enterobacter spp. was promoted by the addition of sorbitol to the media, like as glucose, but $P$. mirabilis failed to grow in sorbitol-supplemented medium (Figure 3A). This suggested that at least four types of bacteria from family Enterobacteriaceae were able to degrade sorbitol. Consistent with this, the concentration of sorbitol in the supernatants of the E. coli, C. farmeri, K. pneumoniae, and Enterobacter spp. cultures was lower than for control medium, but comparable in the supernatants of the P. mirabilis cultures. (Figure 3B). We also found that some Clostridium species (re-classified as Lachnoclostridium [18]) were able to utilize sorbitol and promoted their growth (Figure S3).

We next conducted a gnotobiotic experiment using sorbitol-degrading bacteria and non- sorbitol-degrading bacteria in order to verify that the ability of Enterobacteriaceae to degrade sorbitol helped protect against sorbitol-induced diarrhea. GF mice were inoculated with E. coli as a sorbitol-degrading bacterium and P. mirabilis as a non-sorbitol-degrading bacterium. The mice were allowed access to drinking water containing sorbitol ad libitum. The fecal water content was significantly increased after administration of sorbitol in the GF mice and GF mice inoculated with P. mirabilis, while that in the GF mice inoculated with E. coli did not change when treated with sorbitol (Figure 3C). Representative results regarding the stool properties of each groups after sorbitol treatment are shown in Figure 3D. These results indicated that sorbitol-consuming members of Enterobacteriaceae prevented sorbitol-induced diarrhea. 

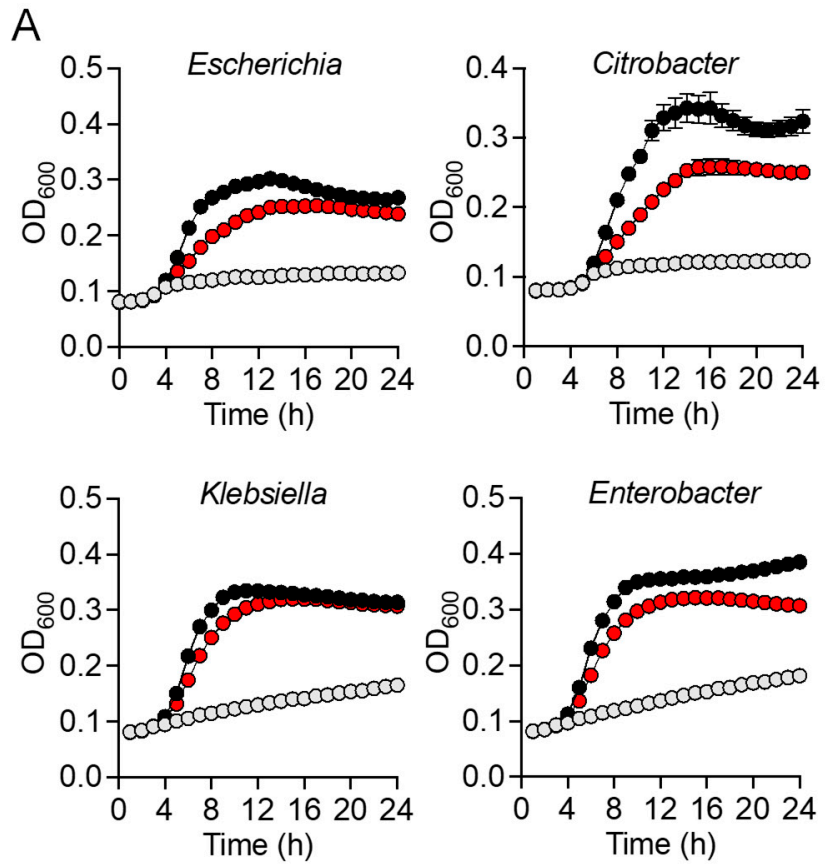

C
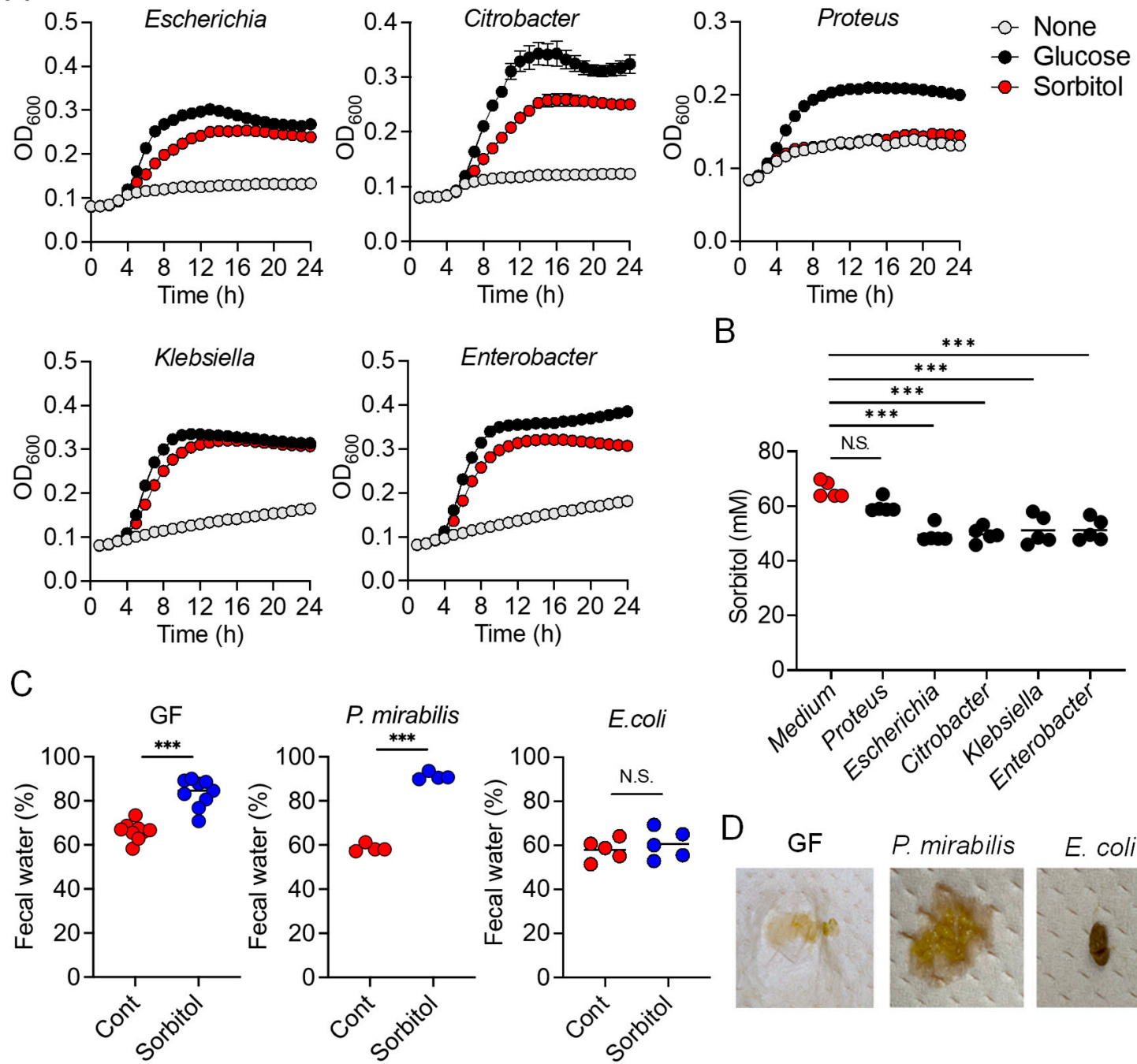

$\mathrm{B}$
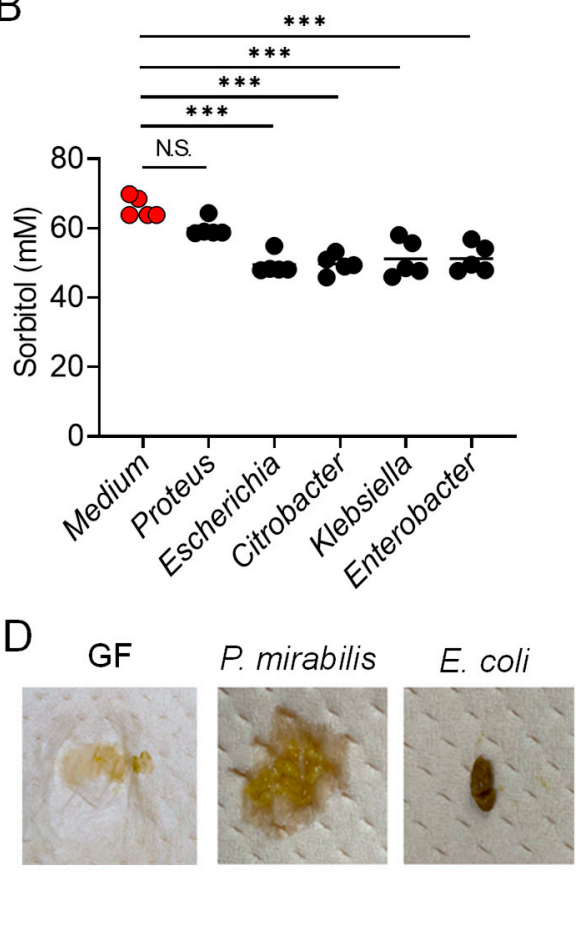

Figure 3. Escherichia coli are Able to Degrade Sorbitol and Suppress Sorbitol-Induced Diarrhea. (A) Each Enterobacteriaceae species was individually grown in M9 medium or M9 medium supplemented with 1\% glucose or sorbitol and optical density (O.D.) at $600 \mathrm{~nm}$ was measured over time. (B) Sorbitol concentrations in $24 \mathrm{~h}$ culture supernatants of each Enterobacteriaceae $(n=5)$. Horizontal bars are mean values. N.S.; not significant, ${ }^{* * *} p<0.001$ compared with the control (Medium) group using Dunnett's t-test. (C,D) IQI Germ-free (GF) mice or GF mice colonized with E. coli or P. mirabilis were provided drinking water containing $5 \%$ sorbitol for 4 days (GF mice, GF + E. coli mice; GF + P. mirabilis mice). (C) Fecal water content at day 0 and 4 after treatment with 5\% sorbitol. Cont: control; 5\%:5\% sorbitol. Each dot represents an individual mouse and the horizontal bars indicate mean values. N.S.; not significant, ${ }^{* * *} p<0.001$ by Welch's $t$-test. (D) Image of representative feces from the gnotobiotic mice given $5 \%$ sorbitol for 4 days.

\subsection{SrlD Was Essential for Suppression of Sorbitol-Induced Diarrhea by E. coli}

To identify the pathway responsible for metabolizing sorbitol in E. coli, we used mutants lacking srlA, srlB, and $\operatorname{srlE}$ genes encoding Enzyme II complex which is involved in sorbitol transport, including, along with $s r l D$, the gene encoding PTS-dependent sorbitol-6phosphate dehydrogenase which generates fructose 6-phosphate for glycolysis (Figure 4A). The $\operatorname{srl} A, s r l B$, and $\operatorname{srl} E$ mutants were able to grow in media containing either glucose or sorbitol as the sole carbon source at rates comparable to those of the wild-type control (Figure $4 \mathrm{~B}$ ). In contrast, the srlD mutant was able to grow in a medium containing glucose as a sole carbon source, but not in a medium containing sorbitol as a sole carbon source (Figure 4B). In addition, sorbitol concentrations in the supernatants of the wild-type and $\operatorname{srl} A, \operatorname{srlB}$, and $\operatorname{srl} E$ mutant strains were lower than that of the control medium (Figure 4C). Meanwhile, sorbitol concentration in the supernatants of the $\operatorname{srlD}$ mutant cultures was 
comparable to that of the control medium (Figure 4C). Consistent with this, the inoculation of GF mice with the wild-type E. coli protected the mice against sorbitol-induced diarrhea, but not with the srlD mutant (Figure 4D). These results suggested that sorbitol metabolism, mediated by the sugar phosphotransferase pathway in E. coli, contributed to the suppression of sorbitol-induced diarrhea.

A

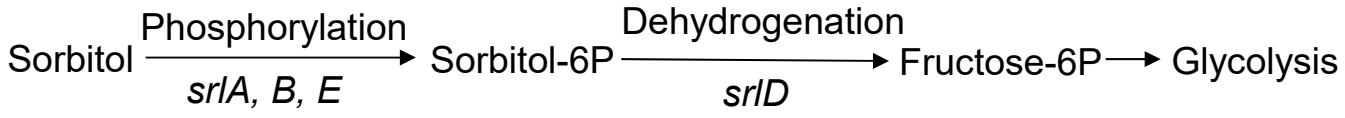

B
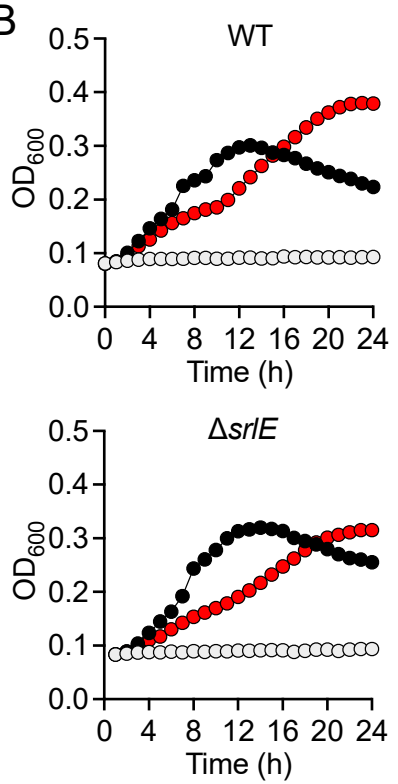

C

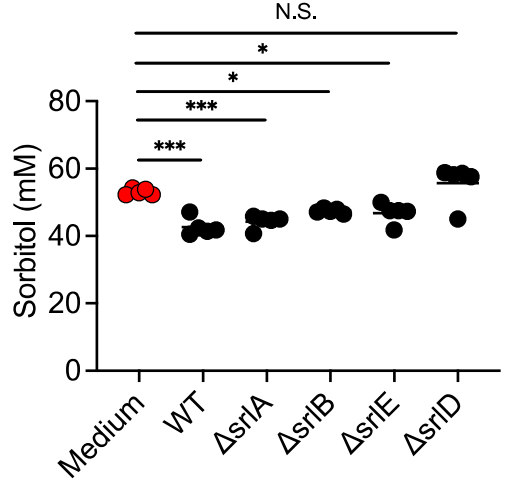

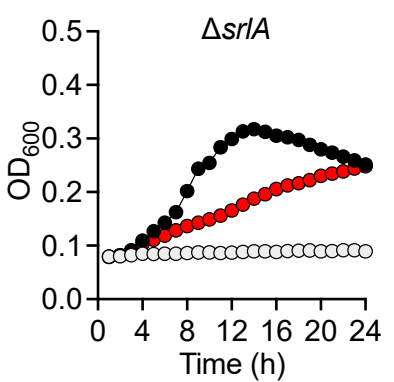
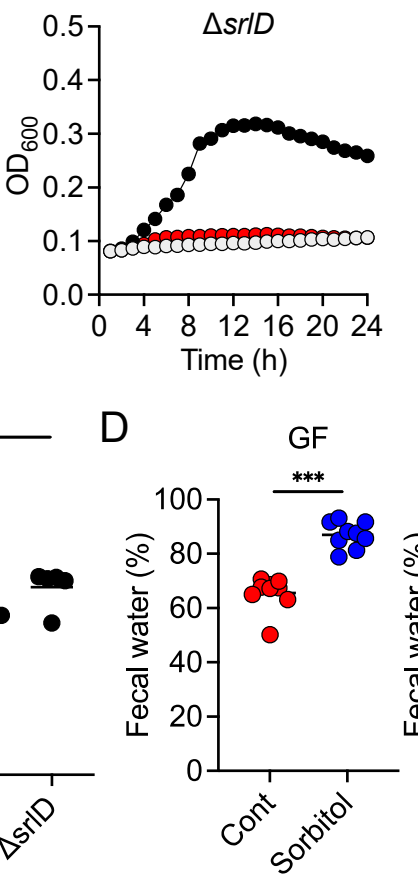

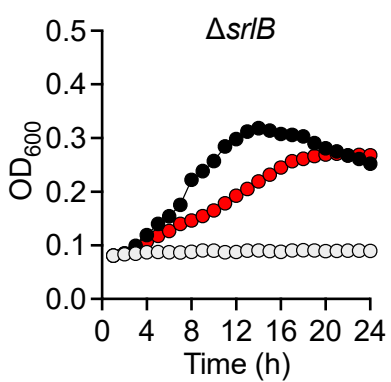

- None

- Glucose

- Sorbitol

Figure 4. srlD Is Required for Suppression of Sorbitol-Induced Diarrhea by Escherichia coli. (A) The pathway of sorbitol metabolism. (B) WT and $s r l A$, srlB, srlE, or srlD mutants of E. coli were grown in M9 medium or M9 medium supplemented with $1 \%$ glucose or sorbitol and optical density (O. D.) at $600 \mathrm{~nm}$ was measured over time. (C) Sorbitol concentration in $24 \mathrm{~h}$ culture supernatants of WT or mutant E. coli strains $(n=5)$. Horizontal bars are mean values. ${ }^{*} p<0.05,{ }^{* * *} p<0.001$, compared with the control (Medium) group using Dunnett's $t$-test. (D) ICR Germ-free (GF) mice or GF mice colonized with WT or srlD mutant of $E$. coli were provided drinking water containing $5 \%$ sorbitol for 4 days. Fecal water content at day 0 and 4 after treatment with $5 \%$ sorbitol was assessed. Each dot represents an individual mouse and the horizontal bars indicate mean values. ${ }^{*} p<0.05,{ }^{* *} p<0.01,{ }^{* * *} p<0.001$ by Welch's $t$-test. WT, wild-type E. coli. N.S., not significant.

\section{Discussion}

In the current study, we demonstrated the protective role of gut bacteria against sugar alcohol-induced diarrhea using both GF mice that lack gut bacteria and mice that had altered gut microbiota as a result treatment with antibiotics. When exposed to sorbitol at a dose that failed to induce diarrhea in SPF mice, GF mice exhibited severe diarrhea 
and significant weight loss. GF mice are known to have higher fecal water content than that of SPF mice under normal conditions, but while the fecal water content of the SPF mice in our study did not change with sorbitol treatment, the fecal water content of the GF mice increased with sorbitol treatment. Since antibiotics such as ampicillin, streptomycin, vancomycin, and erythromycin have different antimicrobial spectra, administration of those antibiotics changed the gut microbiota composition of mice to specific states that were dependent on their antimicrobial spectra. Mice with gut microbiota composition altered by ampicillin and streptomycin treatments exhibited increased susceptibility to sorbitol-induced diarrhea. In comparison, mice with gut microbiota composition that had increased proportions of Enterobacteriaceae and Lachnoclostridium as a result of vancomycin and erythromycin treatment, respectively, did not show an increase in their susceptibility to sorbitol-induced diarrhea. Interestingly, the abundance of Enterobacteriaceae increased in a concentration-dependent manner as sorbitol intake increased. This effect was particularly dramatic in mice that received $10 \%$ sorbitol. This sorbitol-induced growth of Enterobacteriaceae most likely contributed to the transient diarrhea and subsequent disappearance of diarrheal symptoms observed in these mice. Enterobacteriaceae expansion may also represent the mechanism underlying the acquisition of sorbitol tolerance observed in individuals after long-term intake.

At the species level, E. coli constituted approximately $70 \%$ of the gut Enterobacteriaceae population in vancomycin-treated mice with Enterobacter spp. and P. mirabilis accounting for the remaining 30\%. As Enterobacter spp. and P. mirabilis were not able to utilize sorbitol as an energy source, E. coli appears to have been the main contributor to the suppression of sorbitol-induced diarrhea by Enterobacteriaceae. Indeed, the inoculation of GF mice with E. coli alone was sufficient to generate resistance to sorbitol-induced diarrhea.

While the pathways of sorbitol metabolism vary depending on the particular bacterial species, the sugar phosphotransferase system (PTS) is one of the major known pathways. Sorbitol is phosphorylated in this pathway to sorbitol-6-phosphate by a sugar-specific permease, which is a membrane-bound complex known as enzyme II. The sorbitol-6phosphate is subsequently converted to fructose by PTS-dependent dehydrogenase and the fructose is used for glycolysis [19-21]. An E. coli mutant lacking srlD, which encodes the PTS-dependent sorbitol-6-phosphate dehydrogenase, was unable to degrade sorbitol and did not protect mice against sorbitol-induced diarrhea. This indicates the sorbitol degradation by E. coli was dependent on PTS. However, mutants of $\operatorname{srl} A$, srlB, or srlE, which are genes encoding components of the enzyme II complex, were able to utilize sorbitol as an energy source, suggesting that an enzyme II complex-independent pathway for the production of sorbitol-6-phosphate via phosphorylation of sorbitol may exist in E. coli. On the other hand, we also showed that some Clostridium species (re-classified as Lachnoclostridium) were able to utilize sorbitol. However, Clostridium is known to be able to directly metabolize sorbitol to fructose unlike $E$. coli which metabolizes sorbitol to fructose via the PTS pathway [22,23].

Although some strains of $E$. coli can cause infectious and inflammatory diseases in humans, many others live peacefully in human intestines, aiding in digestion and even defending their host against harmful microbes [24]. We found that E. coli was increased and suppressed sorbitol-induced diarrhea after the ingestion of sorbitol. In human adults, E. coli is not the dominant bacteria in the intestine. However, these microbes may maintain a hospitable environment in the gut by preventing the detrimental effects of artificial sweeteners which are widely used in processed foods.

Our current study revealed the relationship between sugar alcohol-induced diarrhea and the gut microbiota, and identified specific gut bacteria that responded to and degraded the sorbitol. However, mannitol and xylitol (which like sorbitol, are poorly-absorbed sugar alcohols) are also widely used as artificial sweeteners in processed foods. As the metabolism of these sugar alcohols by the gut bacteria may differ from that of sorbitol, the species of gut bacteria that protect against diarrhea induced by these sugar alcohols 
may also be different. Further work is needed to clarify whether sugar alcohols other than sorbitol are degraded by different gut microbes.

The gut microbiota has been reported to be associated with various diseases, including some lifestyle-related diseases [25-27]. Accordingly, manipulation of the gut microbiota has received attention for its potential exploitation in supporting human health, such as the use of stool transplantation or metabolites derived from gut microbiota $[28,29]$. Prebiotics are compounds in food that induce the growth or activity of beneficial microorganisms, such as by altering the composition of the gut microbiome [30]. In contrast, dysbiosis is a term for microbial imbalance that may causes negative health symptoms [31]. Dysbiosis can arise from diverse causes, including antibiotic use or consumption of an inappropriate diet [32]. Our current findings regarding the effect of sorbitol on the gut microbiota suggest that artificial sweeteners in processed foods can act as either prebiotics that promote a healthy gut or as inappropriate dietary elements that may cause dysbiosis. We believe our findings provide helpful information elucidating the effects of artificial sweeteners in processed foods on human health via gut microbiota.

Supplementary Materials: The following are available online at https:/ /www.mdpi.com/article/10 .3390/nu13062029/s1, Figure S1. Mannitol (Man)-Induced Diarrhea is Induced by Ampicillin (Amp) Administration; Figure S2. Relative Abundance of Genera of the Order Clostridiales; Figure S3. Some Clostridium species (re-classified as Lachnoclostridium) Are Able to Utilize Sorbitol as a Carbon Source.

Author Contributions: K.H. (Kouya Hattori) and Y.-G.K. conceived the study and designed the experiments; K.H. (Kouya Hattori), M.A., N.S., K.Y. and Y.-G.K. collected samples and conducted the experiments; K.H. (Kouya Hattori), M.A., N.S., K.Y., K.H. (Koji Hase) and Y.-G.K. analyzed the data; K.H. (Kouya Hattori), M.A. and Y.-G.K. prepared the manuscript; and Y.-G.K. supervised the project. All authors have read and agreed to the published version of the manuscript.

Funding: This work was supported by research grants from JSPS KAKENHI (JP17H05068, JP20K21530 and JP20H03490 to Y.-G.K.), AMED (JP18gm6010004h0003 to Y.-G.K.), Takeda Science Foundation (to Y.-G.K.), The Naito Foundation (to Y.-G.K.), Yakult Bio-Science Foundation (to Y.-G.K.), and the Mochida Memorial Foundation for Medical and Pharmaceutical Research (to Y.-G.K.).

Institutional Review Board Statement: The study was conducted according to the guidelines of the Declaration of Helsinki, and approved by the ethics committee of the Keio University (approved number: 17014).

Informed Consent Statement: Not applicable.

Data Availability Statement: The data presented in this study are available on request from the corresponding author.

Acknowledgments: We thank our laboratory members for helpful discussions. Graphical abstract was created using www.biorender.io (accessed on 10 June 2021).

Conflicts of Interest: The authors declare no competing interests.

\section{References}

1. Saha, B.C.; Racine, F.M. Biotechnological production of mannitol and its applications. Appl. Microbiol. Biotechnol. 2011, 89, 879-891. [CrossRef]

2. Silveira, M.; Jonas, R. The biotechnological production of sorbitol. Appl. Microbiol. Biotechnol. 2002, 59, 400-408. [CrossRef] [PubMed]

3. Mattes, R.D.; Popkin, B.M. Nonnutritive sweetener consumption in humans: Effects on appetite and food intake and their putative mechanisms. Am. J. Clin. Nutr. 2008, 89, 1-14. [CrossRef] [PubMed]

4. Corazza, G.R.; Strocchi, A.; Rossi, R.; Sirola, D.; Gasbarrini, G. Sorbitol malabsorption in normal volunteers and in patients with coeliac disease. Gut 1988, 29, 44-48. [CrossRef] [PubMed]

5. Lenhart, A.; Chey, W.D. A Systematic Review of the Effects of Polyols on Gastrointestinal Health and Irritable Bowel Syndrome. Adv. Nutr. 2017, 8, 587-596. [CrossRef]

6. Islam, S. Sorbitol-based osmotic diarrhea: Possible causes and mechanism of prevention investigated in rats. World J. Gastroenterol. 2006, 12, 7635-7641. [CrossRef] 
7. Sonnenburg, E.D.; Sonnenburg, J.L. Starving our Microbial Self: The Deleterious Consequences of a Diet Deficient in MicrobiotaAccessible Carbohydrates. Cell Metab. 2014, 20, 779-786. [CrossRef]

8. Costea, P.I.; Hildebrand, F.; Arumugam, M.; Bäckhed, F.; Blaser, M.J.; Bushman, F.D.; De Vos, W.M.; Ehrlich, S.D.; Fraser, C.M.; Hattori, M.; et al. Enterotypes in the landscape of gut microbial community composition. Nat. Microbiol. 2018, 3, 8-16. [CrossRef]

9. Nishijima, S.; Suda, W.; Oshima, K.; Kim, S.-W.; Hirose, Y.; Morita, H.; Hattori, M. The gut microbiome of healthy Japanese and its microbial and functional uniqueness. DNA Res. 2016, 23, 125-133. [CrossRef]

10. Robertson, J.S. Computer applications in nuclear medicine. J. Chronic Dis. 1966, 19, 443-459. [CrossRef]

11. Ruiz-Ojeda, F.J.; Plaza-Díaz, J.; Sáez-Lara, M.J.; Gil, A. Effects of Sweeteners on the Gut Microbiota: A Review of Experimental Studies and Clinical Trials. Adv. Nutr. 2019, 10, S31-S48. [CrossRef]

12. Plaza-Diaz, J.; Pastor-Villaescusa, B.; Rueda, A.; Abadia-Molina, F.; Ruiz-Ojeda, F.J. Plausible Biological Interactions of Low- and Non-Calorie Sweeteners with the Intestinal Microbiota: An Update of Recent Studies. Nutrients 2020, 12, 1153. [CrossRef]

13. Caporaso, J.G.; Kuczynski, J.; Stombaugh, J.; Bittinger, K.; Bushman, F.D.; Costello, E.K.; Fierer, N.; Peña, A.G.; Goodrich, J.K.; Gordon, J.I.; et al. QIIME Allows Analysis of High-Throughput Community Sequencing data. Nat. Methods 2010, 7, 335-336. [CrossRef] [PubMed]

14. Bolger, A.M.; Lohse, M.; Usadel, B. Trimmomatic: A flexible trimmer for Illumina sequence data. Bioinformatics 2014, 30, 2114-2120. [CrossRef] [PubMed]

15. Tsuda, A.; Suda, W.; Morita, H.; Takanashi, K.; Takagi, A.; Koga, Y.; Hattori, M. Influence of Proton-Pump Inhibitors on the Luminal Microbiota in the Gastrointestinal Tract. Clin. Transl. Gastroenterol. 2015, 6, e89. [CrossRef] [PubMed]

16. Ishizuka, A.; Tomizuka, K.; Aoki, R.; Nishijima, T.; Saito, Y.; Inoue, R.; Ushida, K.; Mawatari, T.; Ikeda, T. Effects of administration of Bifidobacterium animalis subsp. lactis GCL2505 on defecation frequency and bifidobacterial microbiota composition in humans. J. Biosci. Bioeng. 2012, 113, 587-591. [CrossRef]

17. Marley, J.; Lu, M.; Bracken, C. A method for efficient isotopic labeling of recombinant proteins. J. Biomol. NMR 2001, 20, 71-75. [CrossRef]

18. Vacca, M.; Celano, G.; Calabrese, F.M.; Portincasa, P.; Gobbetti, M.; De Angelis, M. The Controversial Role of Human Gut Lachnospiraceae. Microorganisms 2020, 8, 573. [CrossRef]

19. Reizer, J.; Mitchell, W.J.; Minton, N.; Brehm, J.; Reizer, A.; Saier, J.M.H. Proposed Topology of the Glucitol Permeases of Escherichia coli and Clostridium acetobutylicum. Curr. Microbiol. 1996, 33, 331-333. [CrossRef]

20. Yamada, M.; Saier, M.H. Positive and negative regulators for glucitol (gut) operon expression in Escherichia coli. J. Mol. Biol. 1988, 203, 569-583. [CrossRef]

21. Westermayer, S.A.; Fritz, G.; Gutiérrez, J.; Megerle, J.A.; Weißl, M.P.S.; Schnetz, K.; Gerland, U.; Rädler, J.O. Single-cell characterization of metabolic switching in the sugar phosphotransferase system ofEscherichia coli. Mol. Microbiol. 2016, 100, 472-485. [CrossRef] [PubMed]

22. Ng, K.K.; Ye, R.; Wu, X.; Wong, S. Sorbitol dehydrogenase from Bacillus subtilis. Purification, characterization, and gene cloning. J. Biol. Chem. 1992, 267, 24989-24994. [CrossRef]

23. Ye, R.; Wong, S.L. Transcriptional regulation of the Bacillus subtilis glucitol dehydrogenase gene. J. Bacteriol. 1994, 176, 3314-3320. [CrossRef] [PubMed]

24. Velazquez, E.M.; Nguyen, H.; Heasley, K.T.; Saechao, C.H.; Gil, L.M.; Rogers, A.W.L.; Miller, B.M.; Rolston, M.R.; Lopez, C.A.; Litvak, Y.; et al. Endogenous Enterobacteriaceae underlie variation in susceptibility to Salmonella infection. Nat. Microbiol. 2019, 4, 1057-1064. [CrossRef] [PubMed]

25. Tan, J.; McKenzie, C.; Vuillermin, P.J.; Goverse, G.; Vinuesa, C.; Mebius, R.E.; Macia, L.; Mackay, C.R. Dietary Fiber and Bacterial SCFA Enhance Oral Tolerance and Protect against Food Allergy through Diverse Cellular Pathways. Cell Rep. 2016, 15, $2809-2824$. [CrossRef] [PubMed]

26. Koh, A.; De Vadder, F.; Kovatcheva-Datchary, P.; Bäckhed, F. From Dietary Fiber to Host Physiology: Short-Chain Fatty Acids as Key Bacterial Metabolites. Cell 2016, 165, 1332-1345. [CrossRef] [PubMed]

27. Million, M.; Lagier, J.-C.; Yahav, D.; Paul, M. Gut bacterial microbiota and obesity. Clin. Microbiol. Infect. 2013, 19, 305-313. [CrossRef] [PubMed]

28. Kimura, I.; Ozawa, K.; Inoue, D.; Imamura, T.; Kimura, K.; Maeda, T.; Terasawa, K.; Kashihara, D.; Hirano, K.; Tani, T.; et al. The gut microbiota suppresses insulin-mediated fat accumulation via the short-chain fatty acid receptor GPR43. Nat. Commun. 2013, 4, 1829. [CrossRef]

29. Van Nood, E.; Vrieze, A.; Nieuwdorp, M.; Fuentes, S.; Zoetendal, E.G.; De Vos, W.M.; Visser, C.E.; Kuijper, E.; Bartelsman, J.F.W.M.; Tijssen, J.G.P.; et al. Duodenal Infusion of Donor Feces for RecurrentClostridium difficile. New Engl. J. Med. 2013, 368, 407-415. [CrossRef] [PubMed]

30. Sanders, M.E.; Merenstein, D.J.; Reid, G.; Gibson, G.R.; Rastall, R.A. Probiotics and prebiotics in intestinal health and disease: From biology to the clinic. Nat. Rev. Gastroenterol. Hepatol. 2019, 16, 605-616. [CrossRef]

31. Zhang, Y.-J.; Li, S.; Gan, R.-Y.; Zhou, T.; Xu, D.-P.; Li, H.-B. Impacts of Gut Bacteria on Human Health and Diseases. Int. J. Mol. Sci. 2015, 16, 7493-7519. [CrossRef] [PubMed]

32. DeGruttola, A.K.; Low, D.; Mizoguchi, A.; Mizoguchi, E. Current Understanding of Dysbiosis in Disease in Human and Animal Models. Inflamm. Bowel Dis. 2016, 22, 1137-1150. [CrossRef] [PubMed] 